\title{
A model for selecting alternate foot placement during human locomotion
}

\author{
Renato Moraes \\ Universidade de São Paulo, Ribeirão Preto, SP, Brazil
}

\begin{abstract}
The selection of alternate foot placement is based on visual inputs and the prediction of future foot placement combined with a set of internal rules that are guided by three determinants: minimum foot displacement, stability, and maintenance of forward progression. These three determinants are weighted differently, depending on different constraints. The first purpose of this review is to define and provide evidence to support this model. Alternate foot placement response latency appears to vary according to methodological and/or task constraints. The paradigms and latency measurement methods that are used to investigate alternate foot placement may have important implications for alternate foot placement control. Very short latencies to initiate alternate foot placement can be observed when walking on a treadmill, and longer latencies can be observed when walking on the ground. The second purpose of this review is to discuss differences in the latency to initiate alternate foot placement. The data reviewed herein indicate that looking at multiple parameters is important when studying movement selection and planning during human locomotion. Different latencies to select and implement alternate foot placement are part of a continuum that ranges from the most to the least automatic response. Keywords: adaptive locomotion, alternate foot placement, obstacle avoidance, human locomotion, visual information.
\end{abstract}

Received 06 January 2014; received in revised form 23 June 2014; accepted 24 June 2014. Available online 25 November 2014.

\section{Introduction}

Locomotion on even terrain has been extensively studied in recent decades, including investigations of the neural bases of generating basic locomotor patterns. However, activities in daily life require adaptation in basic locomotor patterns to avoid the loss of stability during gait on uneven terrain. Changes in the direction of locomotion, increases in toe clearance to step over obstacles, and the modulation of step length and width are modifications used to adapt and maintain locomotion over uneven terrain. These modifications are referred to as adaptive locomotion. According to Moraes and Gobbi (2008), adaptive locomotion consists of the ability to adjust the basic pattern of locomotion to environmental demands by considering the conditions of the individual and task goals, with the aim of maintaining dynamic stability.

The anticipatory, reactive, and predictive control of balance is used to ensure dynamic stability during

Renato Moraes, School of Physical Education and Sport of Ribeirao Preto, University of São Paulo, and Research Support Center on Chronic-Degenerative Diseases, University of São Paulo. Correspondence regarding this article should be directed to: Renato Moraes, Escola de Educação Física e Esporte de Ribeirão Preto, Universidade de São Paulo, Av. Bandeirantes, 3900, Ribeirão Preto, 14040-907, Brazil. Phone: +55 16 3602-0522. Fax: +55 16 3602-0551. E-mail: renatomoraes@usp.br adaptive locomotion (Patla, 2003). The anticipatory balance control strategy is based on vision and guided by experience to identify potential disruptions in the travel path. Anticipatory modifications are then made to adapt the pattern of locomotion to environmental conditions. The reactive balance control strategy relies on inputs from sensory systems to detect unexpected perturbations in locomotion and is considered the last line of defense against perturbations. If a person raises the lower limb less than the height of an obstacle (i.e., inefficient anticipatory control), then he will stumble, and the only remaining possibility is to use reactive control by means of mono-and polysynaptic reflexes followed by functional voluntary responses to restore and ensure dynamic balance. The predictive balance control strategy is based on experience and allows the individual to predict expected perturbations generated by ongoing voluntary movements. For example, the task of walking while carrying a shopping bag results in destabilizing moments of force in different joints that must be compensated to ensure dynamic stability.

Previous research on adaptive locomotion that involves modulating step length and width focused on studying the implementation of these adjustments when the task was specified by an external cue or defined a priori (Patla, Robinson, Samways, \& Armstrong, 1989; Patla, 1991, 1993; Warren, Young, \& Lee, 1986). 
Changes in the length, width, and height of the step are essential to adapt locomotion to uneven terrain, and these are the mechanisms that are available for the control system to perform the task of avoiding stepping in undesirable areas. Changes in step length or width with the intention of avoiding an undesirable area on the ground (e.g., a water puddle, hole in the ground, or a piece of glass) is called "alternate foot placement," which is considered an anticipatory balance control strategy.

Studies of alternate foot placement have important implications for our understanding of visually controlled locomotion (Patla, Prentice, Rietdyk, Allard, \& Martin, 1999; Moraes, Lewis, \& Patla, 2004; Weerdesteyn, Nienhuis, Hampsink, \& Duysens, 2004; Weerdesteyn, Nienhuis, Mulder, \& Duysens, 2005; Moraes \& Patla, 2006; Moraes, Allard, \& Patla, 2007), with potential to aid the implementation of visually guided adaptive locomotion in legged robots (Lewis, Lee, \& Patla, 2005). Previous studies showed that the selection of alternate foot placement is based on visual inputs and predictions of future foot placement combined with a set of internal rules that are guided by three determinants: minimum foot displacement, stability, and the maintenance of forward progression (Patla et al., 1999; Moraes et al., 2004). The present review defines and provides evidence to support this model.
An issue related to these studies is the latency to select and implement alternate foot placement. Paradigms that are used to investigate alternate foot placement appear to have important implications for alternate foot placement control, in which we can observe very short latencies to initiate alternate foot placement when walking on a treadmill (Weerdesteyn et al., 2004) and longer latencies when walking on the ground (Moraes et al., 2007). The present review also discusses differences in the latency to initiate alternate foot placement. However, before discussing these issues, providing details about the measurements and protocols that are typically found in studies of alternate foot placement is important.

\section{Measurements used in alternate foot placement studies}

Figure 1A illustrates the normal landing position of the right foot relative to an obstacle (i.e., dashed rectangle) that represents an undesirable landing area or area where stepping should be avoided. The choices to avoid an obstacle can be grouped into four options: long, short, lateral, or medial adjustments. The size of the vectors for each option shows the minimum amount of foot displacement that is necessary to clear the obstacle in each direction. This variable is called predicted

\section{A) Alternate Foot Placement Choices}

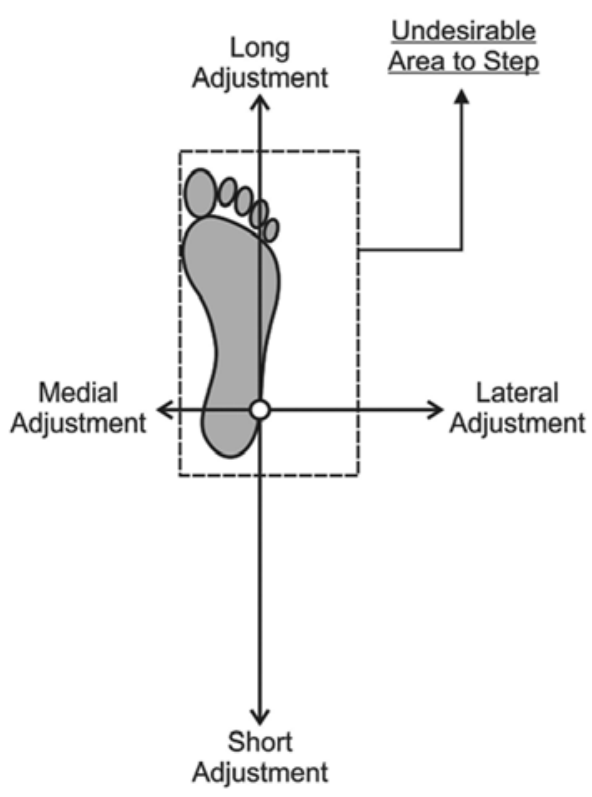

\section{B) Foot Placement Modification Vector}

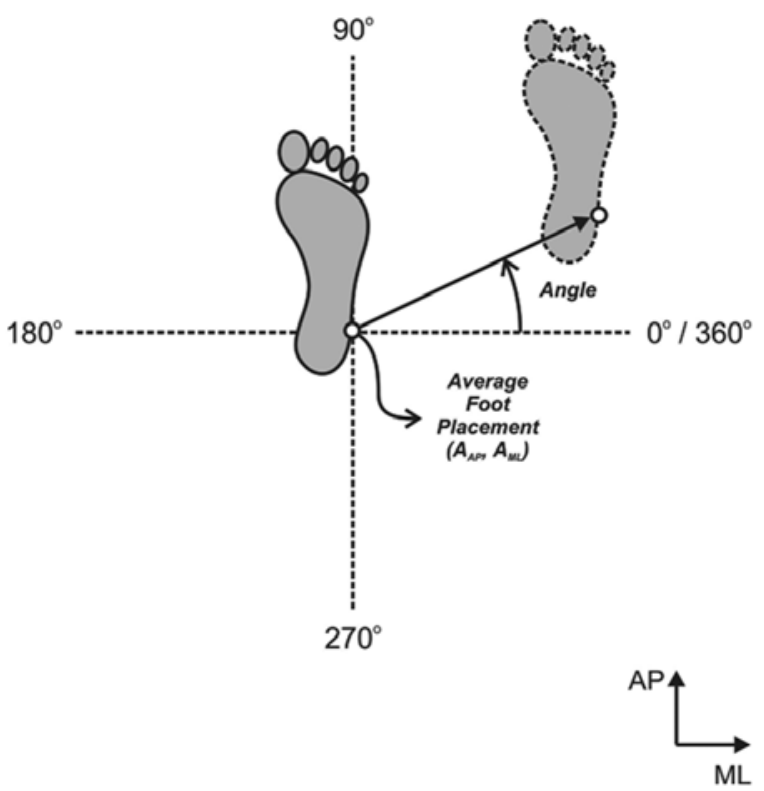

Figure 1. (A) Schematic illustration that shows the normal landing position of the foot relative to the obstacle (undesirable area to step) to avoid when walking. The arrows indicate the direction of the potential choices to avoid the obstacle (i.e., long, short, lateral, and medial). The size of the arrows represents the predicted minimum foot displacement in each direction. In this example, the shortest distance is observed for the medial adjustment, followed by the lateral adjustment. Both long and short adjustments imply much larger displacement of the foot to avoid the obstacle compared with medial and lateral adjustments. (B) The solid-line foot represents the normal landing position of the foot based on the average position of the ankle marker. The dashed-line foot illustrates hypothetical alternate foot placement. The vector that connects the average position of the foot to the alternate foot placement was used to determine the choice made by the participant (based on its angle) and the amount of foot displacement to achieve the alternate foot placement. The white marker located on the posterior region of the foot represents the ankle location (i.e., limb endpoint). AP, anterior-posterior; ML, medial-lateral. 
minimum foot displacement (PMFD). ${ }^{1}$ Details about its computation can be found in Moraes and Patla (2006). To calculate PMFD, having an estimation of average foot placement during normal walking and the dimensions of the foot (i.e., length and width) is necessary.

According to Moraes and Patla (2006), average foot placement is defined as the average coordinates of the marker placed on the lateral malleolus to view the ankle location in tridimensional space. This location is considered the limb endpoint. Typically, the participants walk several times without an obstacle to keep the probability of obstacle appearance low $(20-50 \%$ of the total number of trials). This is important to avoid anticipation during the task. Average foot placement is also used to compute the foot placement modification vector (Figure 1B). The angle of this vector allows the identification of the alternate foot placement choice, especially in studies in which side steps are allowed, whereas the magnitude of this vector indicates the amount of foot displacement from its normal landing position.

\section{Protocols used in alternate foot placement studies}

Studies of alternate foot placement have used different protocols. When walking on a treadmill, a short planar obstacle $(40 \mathrm{~cm}$ length $\times 30 \mathrm{~cm}$ width $\times$ $1.5 \mathrm{~cm}$ height) has been used (Weerdesteyn et al., 2004, 2005; Duysens, Potocanac, Hegeman, Verschueren, \& McFadyen, 2012). This planar obstacle is released on the belt of the treadmill, and participants avoid it by shortening or elongating their step. Side-steps are usually forbidden in these experiments. When walking on the ground, a virtual obstacle has been used to simulate a region on the ground that should be avoided ${ }^{2}$ (Chen, Ashton-Miller, Alexander, \& Schultz, 1994a, b; Patla et al., 1999; Moraes \& Patla, 2006). This virtual obstacle is represented by an illuminated area on the ground. Chen et al. (1994a, b) used a projector and mirror on the ceiling to project the planar obstacle (light band; approximately $3 \mathrm{~cm}$ length $\times 70 \mathrm{~cm}$ width) on the walkway. In these studies, only long and short step strategies are allowed because the walkway and obstacle width have the same dimensions. Patla et al. (1999) used a light-spot projection system that consisted of four lights that were embedded in the middle of the walkway and covered

\footnotetext{
'The use of the word "predicted" to nominate the variable "predicted minimum foot displacement" is not related to the predictive control strategy as defined by Patla (2003). Predicted minimum foot displacement is essentially a mathematical calculation to determine the distance between mean foot location and the edges of the planar obstacle.

${ }^{2}$ Although real obstacles are frequently used in studies of walking on the ground, the major focus of the studies reviewed herein is the behavior of avoidance of planar obstacles because they mimic regions on the ground where people would avoiding stepping. Generally, studies with real obstacles involve major changes in the vertical foot trajectory, whereas the studies reviewed herein involve major changes in the horizontal foot trajectory.
}

with a piece of black cardboard with cuts and a piece of Plexiglas to indicate the illuminated area where the participant should avoid stepping. To allow for different manipulations, a computer monitor substituted this mechanical apparatus with lights (Gonçalves, Moraes, \& Patla, 2004; Greig, Patla, \& Lewis, 2004; Moraes \& Patla, 2006; Moraes et al., 2007). In this case, an obstacle that represents a region to avoid is projected on a screen that is embedded in the walkway. The screen is covered with a piece of Plexiglas to allow normal walking when no obstacle appears. Moraes and Patla (2006) found no behavioral difference when participants avoided a virtual planar obstacle and a real hole of the same length and width. The virtual planar obstacle paradigm appears to be appropriate for research that involves alternate foot placement selection, with the advantage that sidesteps can be performed. Additionally, Moraes et al. (2004) showed that a simple paradigm can be used. In this study, the area to avoid was represented by a region of contrasting color relative to the ground. In addition to these differences, the results of these studies generally provide similar conclusions with regard to alternate foot placement choices (see next section below). However, latency appears to be influenced by these differences in paradigms, especially walking on the ground $v s$. walking on a treadmill, which is discussed in the second part of this review.

\section{Alternate foot placement model}

Movement selection and planning are the main topics of research on motor control. One of the main goals of such research is to understand how specific movements are selected when more than one option allows a goal to be achieved. Some models of upper limb movements have considered multiple factors in movement planning (Rosenbaum, Meulenbroek, Vaughan, \& Jansen, 2001b; Rosenbaum, Meulenbroek, \& Vaughan, 2001a; Patla and Sparrow 2000) instead of only one (Uno, Kawato, \& Suzuki, 1989). Rosenbaum et al. (2001b) proposed a constraint hierarchy model for manual prehension where the end-posture is selected before movement execution. Constraint hierarchy is defined as a list of prioritized factors that are necessary to perform a task. For example, in a simple reaching task, accuracy (i.e., hand-target proximity at the time of movement completion) and movement efficiency (i.e., the exertion of little energy) are the constraints that are considered. The presence of an obstacle and intention to avoid it add a new constraint (i.e., do not collide with the obstacle). The constraints are then considered in a different order: (1) accuracy, (2) hand/arm distance from an obstacle, and (3) efficiency. Therefore, additional constraints redefine the task and weight the priorities differently (i.e., efficiency is less important when avoiding the obstacle). During gait, Moraes et al. (2004) showed that response time constraint affects the priorities that are placed on satisfying various determinants in the choice of alternate foot placement, suggesting that the idea of 
constraint hierarchy can be applied to the alternate foot placement selection.

When a preferred foothold is unavailable during locomotion, selecting, planning, and executing an alternate foot placement are necessary. The selection and implementation of alternate foot placement involve choosing among various options. Patla et al. (1999) originally observed that alternate foot placement selection is not random but rather systematic. Depending on the location of the same target area to be avoided, foot placement selection varies according to the normal landing position of the foot. Thus, visual information about the target area, shape, and size is not sufficient. This information needs to be coupled with prediction of the foot's normal landing position. Patla et al. (1999) proposed that interactions between visual and proprioceptive inputs and step-cycle control are used to estimate the normal landing position of the foot. A mechanism by which this visual and proprioceptive information can be integrated to estimate future foot placement is the forward internal model (Wolpert, Ghahramani, \& Jordan, 1995).
The estimation of the normal landing position is then combined with a set of internal rules that comprise three determinants - minimum foot displacement, stability, and forward progression maintenance-to select the appropriate response (Moraes et al., 2004). As discussed below, these three determinants are weighted differently, depending on timing and spatial constraints (Moraes et al., 2004; Moraes \& Patla, 2006; Moraes et al., 2007). Figure 2 illustrates this model, showing the bases for selection and determinants with gain control. Gain control is needed because, depending on the context, these determinants are weighted differently.

Different studies have shown that minimum foot displacement from its normal landing position is one of the determinants used in the selection of alternate foot placement (Moraes et al., 2004, 2007; Moraes \& Patla, 2006; Weerdesteyn et al., 2005). The prediction of the normal landing position of the foot is used to compute the amount of foot displacement that is required for each alternate foot placement option (i.e., long, short, medial, and lateral). This computation is needed to

\section{Bases for selection}

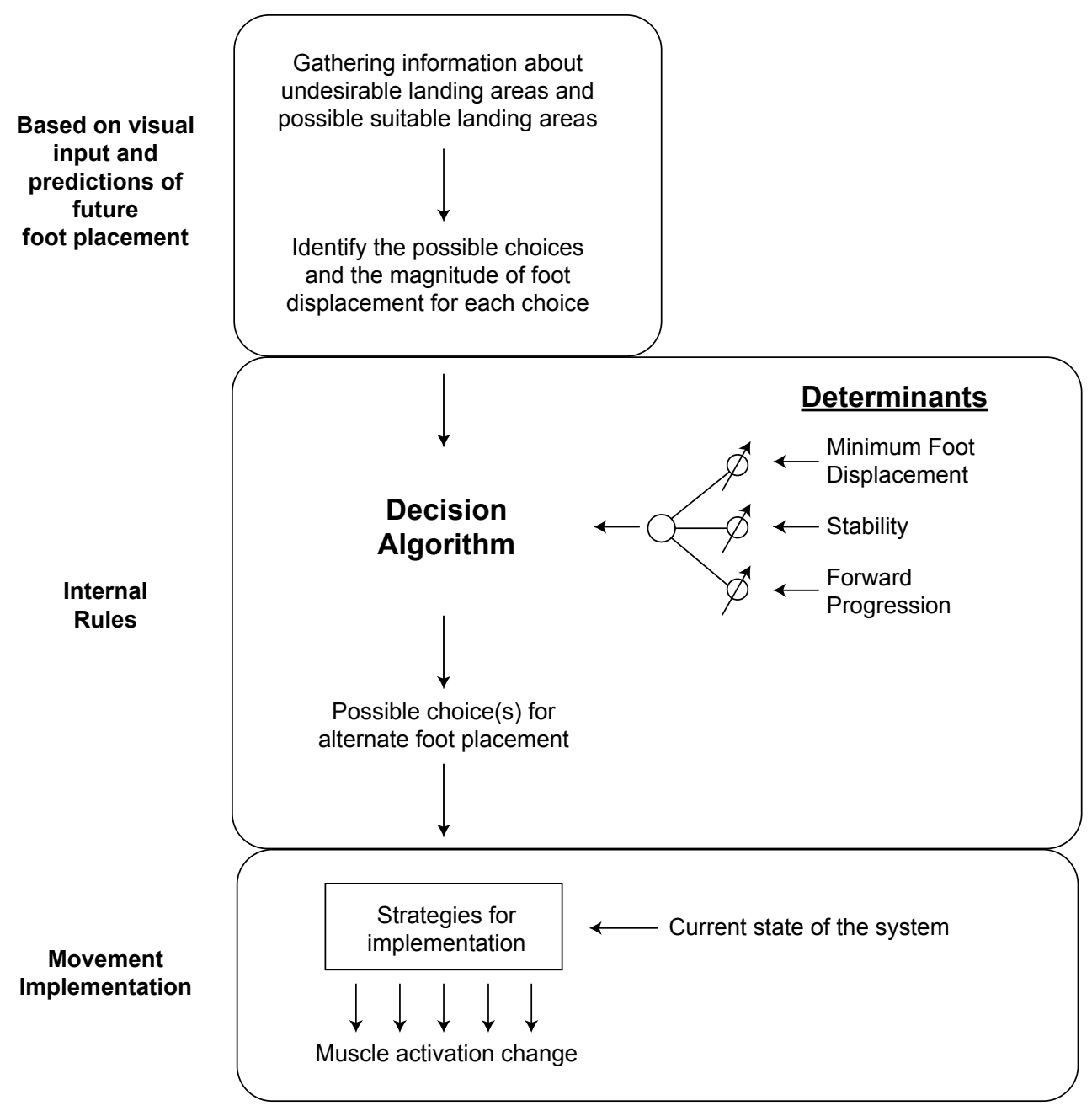

Figure 2. Alternate foot placement model that shows the bases for selection and determinants. 
identify the option that minimizes displacement of the foot. Different studies have shown that dominant choice generally corresponds to the option that minimizes foot displacement (Moraes et al., 2004, 2007; Moraes $\&$ Patla, 2006). The amount of foot displacement is also just sufficient to clear the obstacle because the difference between the magnitude of the foot placement modification vector and PMFD is small.

This preference for minimizing foot displacement appears to be economy-related (i.e., energy cost). This is reasonable because converging evidence suggests that movements are planned to minimize the associated metabolic cost (Cavanagh \& Williams, 1982; Donelan, Kram, \& Kuo, 2001, 2002). Moraes and Patla (2006) monitored the electromyographic activity of the major muscle groups from both the lower limbs and trunk (14 muscles bilaterally) while avoiding real obstacles (i.e., holes on the ground) and virtual planar obstacles. A strong, positive correlation exists between muscle activity and oxygen uptake (Henriksson \& BondePetersen, 1974; Kyröläinen, Belli, \& Komi, 2001; Millet, Perrey, Candau, \& Rouillon, 2002; Praagman, Veeger, Chadwick, Colier, \& van der Helm, 2003; Sengupta \& Das, 2004), and a variable can be calculated that accounts for the percentage change in total muscle activation (PCTMA) for each alternate foot placement choice. Moraes and Patla (2006) found that changes in PCTMA explained $41 \%$ and $35 \%$ of the variability in the magnitude of the foot placement modification vector for virtual and real obstacles, respectively. This small variability, which can be explained by the PCTMA, may be related to the small number of muscles monitored. Additionally, it showed a relationship between changes in muscle activity and foot displacement. This result is consistent with other studies, suggesting that economy is an important parameter in the planning and execution of movements (McNeill Alexander, 2002; Patla \& Sparrow, 2000; Rosenbaum et al., 2001b). More recently, de Boer, Wisse, and van der Helm (2010) used modeling to show that the preference for choosing either the elevating or lowering strategy to regain balance after stumbling over an obstacle is related to recovery cost minimization. Their model showed that limb lowering, which is preferred when obstacle stumbling occurs late during the swing phase (Eng, Winter, \& Patla, 1994), was implemented with smaller torque at the hip joint than when using the limb-elevating strategy over the same swing phase period. Therefore, the choice of strategy reflected the minimization of torque that is required to successfully recover balance after stumbling over an obstacle.

However, the dominant choice is not always the one that minimizes foot displacement, which was first demonstrated by Moraes et al. (2004). These authors proposed that minimum foot displacement from its normal landing position is not the primary determinant but rather one of the determinants used in the decision process. Different studies have shown a preference for long choices over short choices, even when minimum foot displacement favors the short option. Weerdesteyn et al. (2005) showed that older females prefer lengthening their strides over shortening their strides when avoiding planar obstacles during treadmill walking, although the minimum displacement determinant would support a short option. Similarly, Den Otter, Geurts, de Haart, Mulder, and Duysens (2005) found that step lengthening was preferred by individuals who suffered a stroke. Additionally, when the available response time is sufficiently long, there is a preference for step lengthening in young and old adults (Chen et al., 1994b; Weerdesteyn et al., 2005). In young adults, Greig, Patla, and Lewis (2004) found that the switch from the long to the short choice appears to occur when the amount of foot displacement for the long adjustment exceeds the short one by more than $7 \mathrm{~cm}$. Moraes et al. (2007) found that the difference between PMFD for long and short choices was approximately $4 \mathrm{~cm}$, favoring the short option, but the preference for all of the participants was to lengthen their steps.

Two other determinants are also used in the decision-making process: stability requirements and the maintenance of forward progression. Moraes et al. (2007) showed that long and lateral adjustments are preferred over short and medial ones, respectively, because of stability requirements. To reach this conclusion, the margin of dynamic stability (MDS) was used based on extrapolated center of mass (COM) position and the boundaries of the base of support (BOS) as proposed by Hof, Gazendam, and Sinke (2005). For both long and short choices, the extrapolated COM in the anterior-posterior (AP) direction was located away from the BOS, but the extrapolated COM for the long option was closer to the boundaries of the BOS than for the short option. For lateral and medial choices, the extrapolated COM was located inside the BOS in the medial-lateral (ML) direction only for the lateral option. These preferences are based on stability because short adjustments result in unstable movement, attributable to the need to reduce linear body momentum in the AP direction (Patla et al., 1999). If not controlled properly, this forward AP momentum can be converted to clockwise angular momentum in the sagittal plane, which could increase body instability and, in extreme cases, generate a forward fall. Lateral adjustments are preferred because they enlarge the BOS and ensure the projection of the COM within the bounds of the BOS (Moraes et al. 2007).

The maintenance of the forward progression determinant establishes that alternate foot placement that minimizes deviation from moving toward the forward goal is preferred. There is a preference for changes in the plane of progression over changes in the frontal plane (Patla et al., 1999). Moraes et al. (2004) observed that changes in the frontal plane are accompanied by foot placement that is located more forward to minimize deviation from the forward goal. Using a more objective measurement, Moraes et al. (2007) observed decoupling between the foot and COM movement direction 
during medial and lateral alternate foot placements. Specifically, they showed that the COM continued to move forward, whereas the foot moved sideways at the alternate foot placement. Therefore, even for changes in foot placement in the frontal plane, overall body movement is kept in the plane of progression.

When the alternate foot placement must be selected and implemented under temporal constraints (i.e., during the execution of a step) as in the study by Patla et al. (1999), the determinants are satisfied in the following order: (1) minimum foot displacement, (2) stability, and (3) forward progress maintenance. Not all of these determinants are satisfied in all cases when foot placement must be changed quickly. For example, under conditions in which participants stepped medially (Patla et al., 1999), they satisfied the first two determinants but not the latter one. In studies in which the participants could only perform short or long adjustments, exceptions to the minimum foot displacement determinant were also observed, as discussed above. Older females and stroke patients strongly preferred lengthening their step to avoid an obstacle, although step shortening would result in the minimization of foot displacement (Den Otter, Geurts, de Haart, Mulder, \& Duysens, 2005; Weerdesteyn et al., 2005). In these cases, the stability determinant is prioritized. These populations are characterized by problems with stability during locomotion (Bosse, Oberländer, Savelberg, Meijer, Brüggemann, \& Karamanidis, 2012; Balaban \& Tok, 2014). Duysens et al. (2012) also argued that this lengthening preference could be related to the typically observed high success rate for step lengthening compared with step shortening (Weerdesteyn et al., 2005). Moreover, step lengthening provides more time to change the swing limb trajectory (Patla et al., 1999). In contrast, when there are no time or spatial constraints (i.e., obstacle visible from the starting position) for alternating foot placement, as in the study by Moraes et al. (2004), the order of priority changes. The maintenance of forward progression becomes the first determinant, followed by stability and ultimately minimum foot displacement. The maintenance of the forward progression priority is illustrated by the bias toward making long-medial adjustments when the medial choice is the most economical one for the obstacle position tested. Thus, when there is adequate time to plan and implement alternate foot placement, deviations from the travel direction can be minimized through anticipatory control during the approach phase. Therefore, the choice of alternate foot placement results from the search for an optimal solution that satisfies all three determinants together. The gain controls in Figure 2 illustrate this solution.

Although these three determinants proposed by Patla et al. (1999) have been validated in a series of studies conducted by Moraes and collaborators (Moraes et al., 2004, 2007; Moraes \& Patla 2006) and studies by other research groups (Weerdesteyn et al., 2005; Duysens et al., 2012), whether they generalize to different contexts requires further investigation. As discussed above, the absence of temporal constraints enhanced forward progression, which in turn became the dominant determinant followed by stability and lastly energy costs related to the modifications (Moraes et al., 2004). However, when the time available to implement alternate foot placement is reduced (i.e., by forcing the choice to be made and implemented in less than one step duration), the preferred response is boosted (Patla et al., 1999). In contrast, when an unexpected change in obstacle position occurs after the presentation of an initial obstacle, the choice of alternate foot placement is affected (Gonçalves et al., 2004). Figure 3 was adapted from Gonçalves et al. (2004) $)^{3}$. For two obstacle positions, the dominant choices were altered from choices in the frontal plane (i.e., ML choices) for the no-change condition to choices in the sagittal plane (i.e., long/short choices) for the change condition. The bias for long and short modifications observed in the change condition, especially for AP obstacles (i.e., P1, P2, and $\mathrm{P} 3$ ), could be related to the preference for changes in the plane of progression over changes in the frontal plane, as suggested by Patla et al. (1999). Although Patla et al. (1999) made this observation based on conditions in which the amount of foot displacement was the same in both the frontal and sagittal planes, with sudden changes in obstacle position, the control system would prefer changes in the plane of progression because they could be more natural to implement and would facilitate the maintenance of forward progression.

${ }^{3}$ Seven healthy young adults (six females and one male; mean age, $25.1 \pm 2.3$ years; mean height, $1.66 \pm 0.07 \mathrm{~m}$; mean weight, $58.2 \pm 6.1 \mathrm{~kg}$ ) volunteered for this study. The participants were instructed to walk down a 7.3-m-long wooden walkway. A forceplate and liquid crystal display monitor $(38.0 \times 30.0 \mathrm{~cm})$ were embedded in the walkway. The vertical component of the ground reaction force was used to trigger the appearance of a white rectangular planar obstacle on the monitor (threshold > $5 \mathrm{~N}$ ). The planar obstacle appeared at the right heel contact (HC) of the contralateral limb on the forceplate in six different locations (Figure 3 ). The participants then had one step to plan and implement alternate foot placement to avoid stepping on the obstacle. Two experimental conditions were tested: nochange and change. In the no-change condition, the planar obstacle appeared at the right $\mathrm{HC}$ and was kept in the same location throughout the trial. In the no-change condition, the participants performed five trials for each obstacle location, for a total of 30 trials. In the change condition, a planar obstacle appeared at the right $\mathrm{HC}$, but after $100 \mathrm{~ms}$ this first obstacle disappeared and a new planar obstacle simultaneously appeared on the monitor in a different location. For example, the first obstacle could be P1; $100 \mathrm{~ms}$ later, it changed to one of the remaining five obstacle locations. In the change condition, the participants performed one trial for each change in obstacle location. Because there were five changes for each initial obstacle location, the participants performed a total of 30 trials. To reduce the participants' anticipation, the probability of obstacle appearance was set at 0.3 . Therefore, the participants performed 60 experimental trials (30 nochange trials and 30 change trials) and 120 walk through (WT) trials (i.e., without obstacle appearance), for a total of 180 randomized trials. 
Choices were also more distributed in the change condition compared with the no-change condition for the AP obstacles (Figure 3). Moreover, foot displacement increased in the change condition, especially because long and short choices for the AP obstacle imposed the need to increase the amount of foot displacement relative to the normal landing position. Choices in the change condition did not necessarily follow the minimum foot displacement as the major determinant. This result suggests that changes in the obstacle affected the order of priority of determinants to alternate foot placement.

This more distributed pattern contradicts a previous study that reported an increase in the percentage of dominant choice for each foot-obstacle configuration when time pressure increased by making the obstacle to appear $100 \mathrm{~ms}$ after contralateral HC (Patla et al., 1999). The fundamental difference is that in the study by Patla et al. (1999), no previous virtual obstacle was presented during the $100 \mathrm{~ms}$ interval after contralateral HC. This discrepancy in the results suggests that alternate foot placement selection for the first obstacle that was presented at the contralateral $\mathrm{HC}$ may occur during this $100 \mathrm{~ms}$ interval. This helps explain the more distributed pattern because there was a need to rescale or modulate ongoing muscle activity or make a new choice for the new obstacle configuration (new motor plan).

Tseng, Stanhope, and Morton (2009) assessed lower limb responses to lateral shifts in target position during step initiation from a quiet standing position when the target shifted $450 \mathrm{~ms}$ (early), $550 \mathrm{~ms}$ (middle), or 650 ms (late) after initial target presentation. The target always shifted in the lateral direction, and the amount of displacement from its initial position was always $20 \mathrm{~cm}$. This suggests that the participants needed only to add an extra amount of limb displacement to the previously planned movement (rescale/modulate ongoing muscle activity). Additionally, Patla, Beuter, and Prentice (1991) observed a two-stage correction to avoid a vertical obstacle that was raised as the individuals lifted their foot. In this case, the first and generic response, which was adequate for low and high obstacles, occurred $122 \mathrm{~ms}$ after triggering the unexpected obstacle. The second response occurred $158 \mathrm{~ms}$ after the first response and corresponded to fine-tuning of the limb trajectory as a function of obstacle height. This suggests that individuals plan for an initial response that would be adequate for the highest obstacle, but they could change this plan after a short time to make it adequate for the correct obstacle height (new motor plan after movement initiation). Changes in foot placement after changes in the obstacle could be attributable to both explanations (i.e., rescaling/modulating ongoing muscle activity and a new motor plan).

Notably, the stability and forward progression determinants were unaffected by obstacle changes (Gonçalves et al., 2004). Although the minimization of foot displacement was not necessarily maintained when avoiding the planar obstacle, the stability and forward

\section{AP Obstacles}

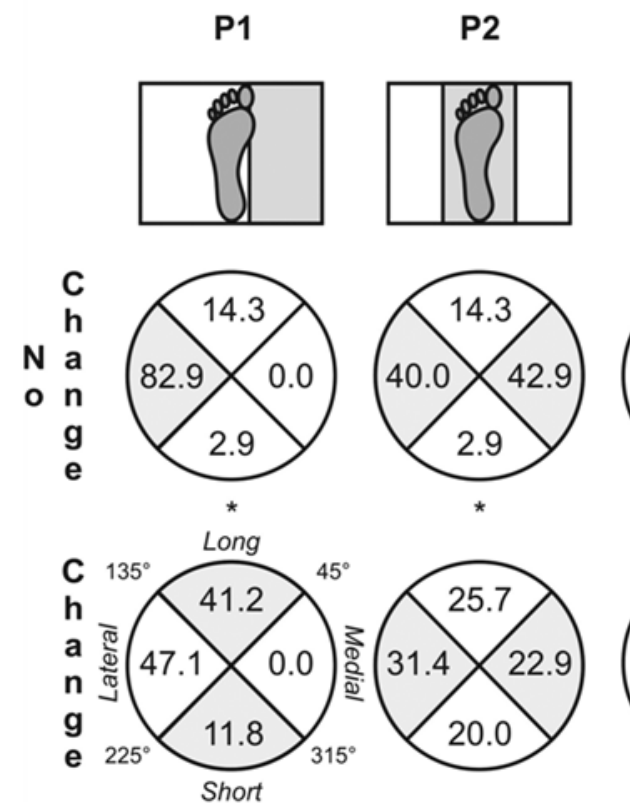

P3
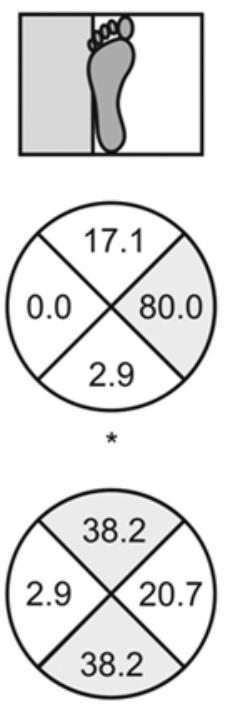

P4

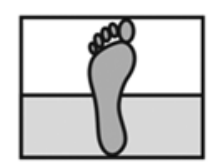

85.7

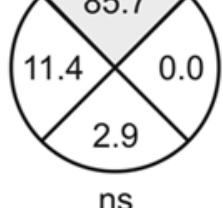

ns

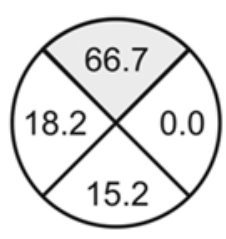

\section{Obstacles}

P5

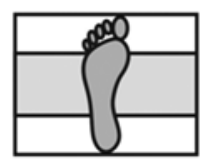

37.1
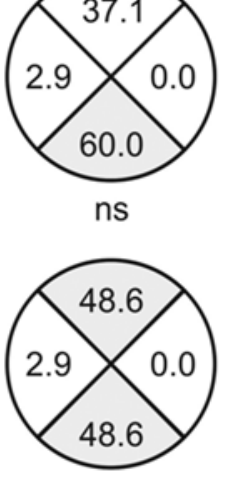

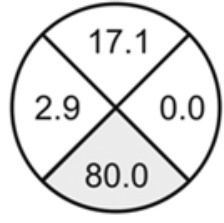

$\mathrm{nS}$

P6

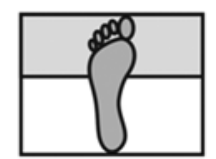

17.1

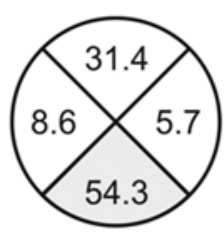

Figure 3. Percentage of alternate foot placement choices made by participants in both the no-change and change conditions. The first row shows the obstacle positions with a representation of the normal foot landing position. Obstacles are shown in gray. The shaded quarters within each circle represent the dominant choice $(>50 \%)$. When more than one quarter is shaded, there is no dominant choice, but the sum of them represents the direction in which the majority of the choices were made. In the change condition, when referring to obstacle positions, it is the final obstacle position that is being considered for data presentation and analyses. The data for the change condition were collapsed across final obstacle position (see footnote 3 for details about the experimental setup). These data are based on a sample of seven healthy young adults. ${ }^{*} p$ $<.05$. ns, nonsignificant. (Adapted from Gonçalves et al., 2004.) 
progression determinants were kept the same in both the no-change and change conditions. The system seems to prefer to be less economical but able to maintain the entire body in condition to keep walking straight ahead. Therefore, under time constraints after an initial obstacle presentation, the contribution (or weight) of stability and forward progression increases, based on the decision algorithm proposed by Moraes et al. (2004) and expanded on in the present review, whereas the minimization of foot displacement becomes less important (i.e., reduced weight).

This result is consistent with Rosenbaum et al. (2001b), who proposed the notion of a constraint hierarchy. The idea of a constraint hierarchy is important because it assumes multiple constraints rather than just one, as in previous prevailing ideas in motor control research (Uno et al., 1989). Although the constraint hierarchy was originally conceptualized for the planning of movement with a specific goal, this idea may also be adapted to alternate foot placement selection. In fact, Bahrami and Patla $(2005)^{4}$ modeled alternate foot placement selection based on these three determinants by properly weighting them according to task priorities, such as those used in the constraint hierarchy. Based on this model, they were able to correctly predict $85 \%$ of the choices. Then, depending on the goal of the task and context of its execution, some aspects were prioritized wherever others became less important.

\section{Latency}

Projections from the ventral premotor cortex (PMv) to primary motor cortex (M1) have been proposed to inhibit the activity of the latter region when an action-relevant stimulus changes during the course of movement preparation or execution (Mars, Piekema, Coles, Hulstijn, \& Toni, 2007; Buch, Mars, Boorman, \& Rushworth, 2010). This process has been termed "action reprogramming" (Neubert, Mars, Buch, Olivier, \& Rushworth, 2010). Action-relevant stimuli are typically manipulated by having the participant reach and grasp or point to a target. Before or during movement execution, the object to be grasped or target to be pointed to has its location changed unexpectedly. Several studies have shown that the latency of this action reprogramming after movement onset, especially for pointing, is performed very quickly, with latencies as low as 100 ms (Day \& Brown, 2001; Fautrelle, Prablanc, Berret, Ballay, \& Bonnetblanc, 2010; Paulignan, MacKenzie, Marteniuk, \& Jeannerod, 1991), suggesting

\footnotetext{
${ }^{4}$ They developed a model based on fuzzy rules to simulate path planning in "an environment with disconnected footplacement sectors (islands)." The model was based on validity indices and penalty functions, with values varying between 0 and 1. A suitability index was computed as the product of validity indices and penalty functions for each reachable island. The island whose suitability index was maximized was chosen as the next step location. Data from six individuals were used for model validation.
}

the existence of a subcortical pathway. Some authors have suggested the existence of an "automatic pilot" that would be responsible for such very fast responses (Pisella et al., 2000). Day and Lyon (2000) found that when participants were required to respond to an unexpected change in target location with a movement in the opposite direction, the initial component of the response was always in the direction of the new target location. Only after this initially wrong movement was the direction corrected to the opposite side of the new target. Perfiliev, Isa, Johnels, Steg, and Wessberg (2010) suggested that an innate neuronal network would be responsible for controlling goal-directed arm movement toward a target, with latencies ranging from 90 to 110 ms. They tested humans, cats, kittens, and monkeys in a similar task of reaching for an object that moved either to the right or left. Right arm/limb movement toward the target was naturally coupled to the object movement to the right and vice versa for object movement to the left. These results were consistent for mature animals (cats and monkeys) and kittens that do not yet have a mature motor cortex.

Very fast corrections to ongoing movement for the lower limbs during gait or step tasks have also been found (Weerdesteyn et al., 2004; Reynolds and Day, 2005). Weerdesteyn et al. (2004) found a latency of 122 $\mathrm{ms}$ for avoiding an obstacle while walking on a treadmill. During a step task, Reynolds and Day (2005) measured latencies that ranged from 114 to $121 \mathrm{~ms}$ for lower limb frontal plane adjustments. Patla et al. (1991) found latencies of $122 \mathrm{~ms}$ for avoiding an unexpected vertical obstacle after step initiation on the ground. Altogether, these studies have been used to support the existence of a similar, fast subcortical pathway for the lower limbs. However, Moraes et al. (2007) observed long latencies (292 ms) in an obstacle avoidance task when a region on the ground had to be avoided. They argued that, in previous studies, the obstacle/target moved during the task. Because of this dynamic characteristic of the task, the participants had to react faster to respond properly, whereas in the static context of Moraes et al., more time was available to plan and implement the response. Moraes et al. (2007) suggested that the participants preprogrammed the movement less in the dynamic context and relied more on visual feedback during foot adjustment when walking on the treadmill.

One way to test this hypothesis is to rapidly change the position of the obstacle after its initial presentation. This allows studying this possible effect of a dynamic context when walking on the ground. If individuals rely more on visual feedback during foot adjustment, then a reduction of latency would be observed after changing obstacle location. Gonçalves et al. (2004) performed a study in which an unexpected change in obstacle position occurred $100 \mathrm{~ms}$ after presentation of the obstacle in an initial position. These data were reanalyzed and are presented in Figure 4.

The change in obstacle position resulted in a faster response than the one observed in the no-change condition. 

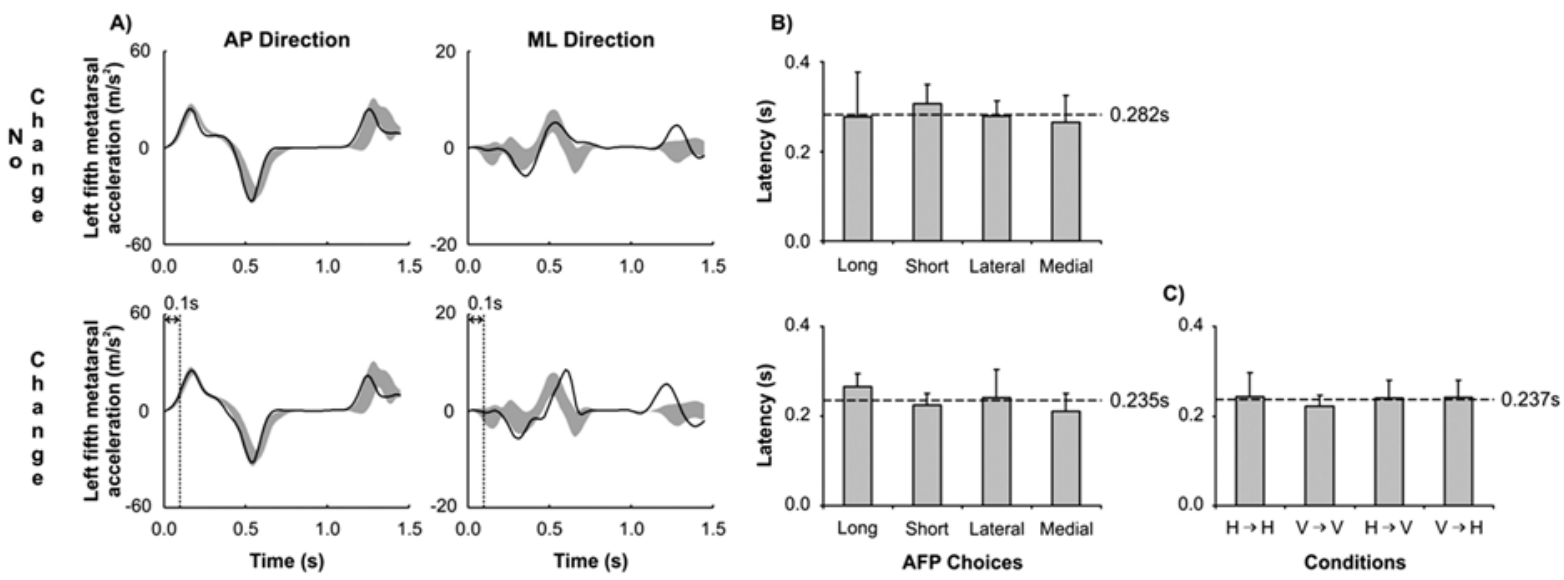

Figure 4. (A) Time histories of left fifth metatarsal linear acceleration that show the mean \pm 2 standard deviations (shaded area) for the AP (left) and ML (right) directions. The top row shows the results for the no-change condition, and the bottom row shows the results for the change condition. Two illustrative trials of both the short and lateral choices are also shown (solid line). The point where the solid line deviates from the shaded area is defined as the onset time of limb trajectory change (i.e., latency). The data were aligned (time $=0 \mathrm{~s}$ ) based on right heel-contact on the force plate, which was the trigger for obstacle appearance. The dashed vertical line in the bottom row indicates the instant the obstacle changed (i.e., $100 \mathrm{~ms}$ or .1 s). For the AP direction, the deviation below the shaded area represents a short choice and vice versa for a deviation above the shaded area (not shown). For the ML direction, the deviation below the shaded area represents a lateral choice and vice versa for a deviation above the shaded area (not shown). (B) The bar graphs show the means and standard deviations for latency for all of the alternate foot placement choices (long, short, lateral, and medial) in both the no-change (top) and change (bottom) conditions. (C) The bar graph shows the means and standard deviations for latency for the change in orientation/position. The change in orientation involved two conditions: AP $\rightarrow \mathrm{ML}$ and $\mathrm{ML} \rightarrow \mathrm{AP}$. The change in position involved two other conditions: $\mathrm{AP} \rightarrow \mathrm{AP}$ and $\mathrm{ML} \rightarrow \mathrm{ML}$. The horizontal dashed lines in $\mathrm{B}$ and $\mathrm{C}$ represent the average values across all choices and conditions illustrated in each graph. (Adapted from Gonçalves et al., 2004)

For latency, one-way analyses of variance (ANOVAs) for both the no-change and change conditions did not reveal a main effect of alternate foot placement choice (Figure 4B). The latencies were then collapsed for subsequent statistical analyses. A paired $t$-test was used to compare the no-change and change conditions, revealing a statistically significant difference between these conditions $\left(t_{6}=2.871\right.$, $p=.028)$. In the no-change condition, the mean latency was higher $(.282 \pm .010 \mathrm{~s})$ than in the change condition $(.235 \pm .012 \mathrm{~s})$. In the change condition, the latency relative to the change in position was also analyzed. The one-way repeated-measures ANOVA (position change) did not reveal any significant effect (Figure 4C). The overall latency was $.237 \pm .012 \mathrm{~s}$. The latency was the same regardless of whether there was a change in obstacle position/orientation in the change trials.

These results support the hypothesis that obstacle changes accelerate alternate foot placement choice and implementation. In the no-change condition, the latency was close to the results obtained by Moraes et al. (2007; i.e., $292 \mathrm{~ms}$ ). However, these high values are in disagreement with other studies that used a similar task (Weerdesteyn et al., 2004; Reynolds \& Day, 2005). The high latency values in the study by Gonçalves et al. may reflect methodological differences or cortical involvement in the selection of alternate foot placement, as proposed by Moraes et al. (2007). Before discussing possible cortical involvement, considering some methodological differences is necessary, especially with regard to the study by Weerdesteyn et al. (2004). First, when walking on a treadmill, the participants may be forced to make faster adjustments compared with overground walking. As reported by Moraes et al. (2007), the participants slowed down in the adaptive step, which gave them extra time to decide and implement appropriate alternate foot placement. The deliberate strategy of slowing down during overground walking could be used to acquire the necessary information about obstacle location and limb position to preprogram the alternate foot placement. Second, obstacle size was larger in the study by Weerdesteyn et al.; combined with the treadmill belt, constant movement may have created a scenario in which the participants had to respond very quickly to successfully avoid the obstacle. In this case, the participants could preprogram their movements less and rely on online sensory information more to control limb trajectory. Third, ipsilateral foot acceleration data were lined up with contralateral HC (i.e., the stimulus used to trigger obstacle appearance). This may be inconvenient because the expected short latencies (i.e., $120 \mathrm{~ms}$ ) will likely fall within the period when the ipsilateral limb is lifting off. Additionally, Oostwoud Wijdenes, Brenner, and Smeets (2011) suggested that using a fixed threshold to compute latency (as was the case for the studies by both Moraes et al. and Gonçalves et al.) may not be appropriate because low-intensity responses would be considered to have occurred later in time. This latter issue should be further investigated in future studies by combining the analysis of the initial intensity response (i.e., acceleration peak after obstacle appearance) and the latency, as suggested by Veerman, Brenner, and Smeets (2008).

As suggested by Moraes et al. (2007), because of the more dynamic nature of the task, participants have 
to preprogram less of the movement and rely more on visual feedback during foot adjustment while walking on a treadmill compared with overground walking. As expected, in a more dynamic context, which was the case in the change condition, the latency was reduced to $235 \mathrm{~ms}$, although this value is still high compared with the $120 \mathrm{~ms}$ that was obtained in previous studies. This reduction of the latency in the change condition may reflect the need for a more urgent response because of the initial process of body weight transfer from the contralateral to ipsilateral foot. The implementation of alternate foot placement then must be accelerated to avoid a loss of balance that could result in a fall or contact with the virtual planar obstacle. In this case, alternate foot placement could be less preprogrammed, and visual feedback could be used to drive foot placement in the intended location. However, the change condition was not characterized by a very dynamic context, demonstrated by the high latency compared with previous studies that used more dynamic contexts (Weerdesteyn et al., 2004).

Because the latency was still high in the change condition and close to typical visual reaction time, cortical pathways may be involved, as suggested by Moraes et al. (2007), even in the more dynamic context. In fact, these different latency values may be on a continuum that ranges from the most to the least automatic response (Day \& Lyon 2000).

\section{Final considerations}

The studies reviewed herein have an important impact on modeling and applications in robotics. Such studies may aid in visually guided adaptive locomotion in legged robots, particularly in making robots more adaptable to cluttered terrain by allowing them to search for and select the options that satisfy these determinants. Theoretically, the data reviewed herein indicate that looking at multiple parameters is important when studying movement selection and planning during human locomotion. Finally, different latencies to select and implement alternate foot placement are on a continuum that ranges from the most to the least automatic response.

\section{References}

Bahrami, F., \& Patla, A. E. (2005). Alternate foot placement in the presence of a planar obstacle: model development. Gait and Posture, 21(Suppl. 1), S137.

Balaban, B., \& Tok, F. (2014). Gait disturbances in patients with stroke. Physical Medicine and Rehabilitation, in press, doi: 10.1016/j.pmrj.2013.12.017

Buch, E. R., Mars, R. B., Boorman, E. D., \& Rushworth, M. F. (2010). A network centered on ventral premotor cortex exerts both facilitatory and inhibitory control over primary motor cortex during action reprogramming. Journal of Neuroscience, 30(4), 1395-1401.

Bosse, I., Oberländer, K. D., Savelberg, H. H., Meijer, K., Brüggemann, G. P., \& Karamanidis, K. (2012). Dynamic stability control in younger and older adults during stair descent. Human Movement Science, 31(6), 1560-1570.

Cavanagh, P. R., \& Williams, K. R. (1982). The effect of stride length variation on oxygen uptake during distance running. Medicine and Science in Sports and Exercise, 14(1), 30-35.
Chen, H. C., Ashton-Miller, J. A., Alexander, N. B., \& Schultz, A. B. (1994a). Effects of age and available response time on ability to step over an obstacle. Journal of Gerontology. Series A, Biological Sciences and Medical Sciences, 49(5), M227-M233.

Chen, H. C., Ashton-Miller, J. A., Alexander, N. B., \& Schultz, A. B. (1994b). Age effects on strategies used to avoid obstacles. Gait and Posture, 2, 139-146.

Day, B. L., \& Lyon, I. N. (2000). Voluntary modification of automatic arm movements evoked by motion of a visual target. Experimental Brain Research, 130, 159-168.

Day, B. L, \& Brown, P. (2001). Evidence for subcortical involvement in the visual control of human reaching. Brain, 124, 1832-1840.

de Boer, T., Wisse, M., \& van der Helm, F. C. T. (2010). Mechanical analysis of the preferred strategy selection in human stumble recovery. Journal of Biomechanical Engineering, 132(7), 071012.

Den Otter, A. R., Geurts, A. C. H., de Haart, M., Mulder, T., \& Duysens, J. (2005). Step characteristics during obstacle avoidance in hemiplegic stroke. Experimental Brain Research, 161, 180-192.

Donelan, J. M., Kram, R., \& Kuo, A. D. (2001). Mechanical and metabolic determinants of the preferred step width in human walking. Proceedings of the Royal Society B: Biological Sciences, 268, 1985-1992.

Donelan, J. M., Kram, R., \& Kuo, A. D. (2002). Mechanical work for step-to-step transitions is a major determinant of the metabolic cost of human walking. Journal of Experimental Biology, 205, $3717-$ 3727 .

Duysens, J., Potocanac, Z., Hegeman, J., Verschueren, S., \& McFadyen, B. J. (2012). Split-second decisions on a split belt: does simulated limping affect obstacle avoidance? Experimental Brain Research, 223(1), 33-42.

Eng, J. J., Winter, D. A., \& Patla, A. E. (1994). Strategies for recovery from a trip in early and late swing during human walking. Experimental Brain Research, 102(2), 339-349.

Fautrelle, L., Prablanc, C., Berret, B., Ballay, Y., \& Bonnetblanc, F. (2010). Pointing to double-step visual stimuli from a standing position: very short latency (express) corrections are observed in upper and lower limbs and may not require cortical involvement. Neuroscience, 169, 697-705.

Gonçalves, C. T., Moraes, R., \& Patla, A. E. (2004). Amending foot placement during a step to sudden change in obstacle location and orientation. Proceedings of the $13^{\text {th }}$ Biennial Conference for the Canadian Society for Biomechanics. Halifax.

Greig, M. A., Patla, A. E., \& Lewis, A. M. (2004). Characterizing step width and step length adjustments for the implementation in robotic locomotion control. Proceedings of the $13^{\text {th }}$ Biennial Conference for the Canadian Society for Biomechanics. Halifax.

Henriksson, J., \& Bonde-Petersen, F. (1974). Integrated electromyography of quadriceps femoris muscle at different exercise intensities. Journal of Applied Physiology, 36(2), 218220.

Hof, A. L., Gazendam, M. G. J., \& Sinke, W. E. (2005). The condition for dynamic stability. Journal of Biomechanics, 38, 1-8.

Kyröläinen, H., Belli, A., \& Komi, P. V. (2001). Biomechanical factors affecting running economy. Medicine and Science in Sports and Exercise, 33(8), 1330-1337.

Lewis, M. A., Lee, H., \& Patla, A. (2005). Foot placement selection using non-geometric visual properties. International Journal of Robotics Research, 24, 553-561.

Mars, R. B., Piekema, C., Coles, M. G., Hulstijn, W., \& Toni, I. (2007). On the programming and reprogramming of actions. Cerebral Cortex, 17, 2972-2979.

McNeill Alexander, R. (2002). Energetics and optimization of human walking and running: the 2000 Raymond Pearl Memorial Lecture. American Journal of Human Biology, 14, 641-648.

Millet, G. Y., Perrey, S., Candau, R., \& Rouillon, J. D. (2002). Relationships between aerobic energy cost, performance, and kinematic parameters in roller ski skating. International Journal of Sports Medicine, 23, 191-195.

Moraes, R., \& Gobbi, L. T. B. (2008). Controle visual da locomoção adaptativa e da mobilidade em indivíduos idosos. In: U.C. Corrêa (Ed.), Pesquisa em comportamento motor: a intervenção profissional em perspectiva (pp. 42-60). São Paulo: EFP/EEFEUSP.

Moraes, R., \& Patla, A. E. (2006). Determinants guiding alternate foot placement selection and the behavioral responses are similar when avoiding a real or a virtual obstacle. Experimental Brain Research, $171,497-510$

Moraes, R., Allard, F., \& Patla, A. E. (2007). Validating determinants for an alternate foot placement selection algorithm during human 
locomotion in cluttered terrain. Journal of Neurophysiology, 98, 1928-1940.

Moraes, R., Lewis, M. A., \& Patla, A. E. (2004). Strategies and determinants for selection of alternate foot placement during human locomotion: influence of spatial and temporal constraints. Experimental Brain Research, 159, 1-13.

Neubert, F., Mars, R. B., Buch, E. R., Olivier, E., \& Rushworth, M. F. (2010). Cortical and subcortical interactions during action reprogramming and their related white matter pathways. Proceedings of the National Academy of Sciences of the United States of America, 107(30), 13240-13245.

Oostwoud Wijdenes, L., Brenner, E., \& Smeets, J. B. J. (2011). Fast and fine-tuned corrections when the target of a hand movement is displaced. Experimental Brain Research, 214, 453-462.

Patla, A. E. (1991). Visual control of locomotion. In: A. E. Patla (Ed.), Adaptability of human gait: implications for the control of locomotion (pp. 55-97). Amsterdam: Elsevier Science Publishers.

Patla, A. E. (1993). Age-related changes in visually guided locomotion over different terrains: major issues. In: G. E. Stelmach, \& V. Hömberg (Eds.), Sensorimotor impairment in the elderly (pp. 231252). Dordrecht: Kluwer Academic Publishers.

Patla, A. E. (2003). Strategies for dynamic stability during adaptive human locomotion. IEEE Engineering in Medicine and Biology Magazine, 22(2), 48-52.

Patla, A. E., Beuter, A., \& Prentice, S. (1991). A two stage correction of limb trajectory to avoid obstacles during stepping. Neuroscience Research Communications, 8(3), 153-159.

Patla, A. E., Robinson, C., Samways, M., \& Armstrong, C. J. (1989). Visual control of step length during overground locomotion: taskspecific modulation of the locomotor synergy. Journal of Experimental Psychology: Human Perception and Performance, 15(3), 603-617.

Patla, A. E., Prentice, S. D., Rietdyk, S., Allard, F., \& Martin, C. (1999). What guides the selection of alternate foot placement during locomotion in humans. Experimental Brain Research, 128, 441-450.

Patla, A. E., \& Sparrow, W. A. (2000). Factors that have shaped human locomotor structure and behavior: the "joules" in the crown. In: W. A. Sparrow (Ed.), Energetics of human activity (pp. 43-65). Champaign, IL: Human Kinetics.

Paulignan, Y., MacKenzie, C., Marteniuk, R., \& Jeannerod, M. (1991). Selective perturbation of visual input during prehension movements: 1. The effects of changing object position. Experimental Brain Research, 83, 502-512.

Perfiliev, S., Isa, T., Johnels, B., Steg, G., \& Wessberg, J. (2010). Reflexive limb selection and control of reach direction to moving targets in cats, monkeys, and humans. Journal of Neurophysiology, 104, 2423-2432.

Pisella, L., Gréa, H., Tilikete, C., Vighetto, A., Desmurget, M., Rode, G., \& Rossetti, Y. (2000). An "automatic pilot" for the hand in human posterior parietal cortex: toward reinterpreting optic ataxia. Nature Neuroscience, 3(7), 729-736.

Praagman, M., Veeger, H. E., Chadwick, E. K., Colier, W. N., \& van der Helm, F. C. (2003). Muscle oxygen consumption, determined by NIRS, in relation to external force and EMG. Journal of Biomechanics, 36, 905-912.

Reynolds, R. F., \& Day, B. L. (2005). Rapid visuo-motor processes drive the leg regardless of balance constraints. Current Biology, 15(2), R48-R49.

Rosenbaum, D. A., Meulenbroek, R. J., Vaughan, J., \& Jansen, C. (2001). Posture-based motion planning: applications to grasping. Psychological Review, 108(4), 709-734.

Rosenbaum, D. A., Meulenbroek, R. J., \& Vaughan, J. (2001). Planning reaching and grasping movements: theoretical premises and practical implications. Motor Control, 2, 99-115.

Sengupta, A. K., \& Das, B. (2004). Determination of worker physiological cost in workspace reach envelopes. Ergonomics, 47(3), 330-342.

Tseng, S. C., Stanhope, S. J., \& Morton, S. M. (2009). Impaired reactive stepping adjustments in older adults. Journal of Gerontology. Series A, Biological Sciences and Medical Sciences, 64A, 807-815.

Uno, Y., Kawato, M., \& Suzuki, R. (1989). Formation and control of optimal trajectory in human multijoint arm movement minimum torque-change model. Biological Cybernetics, 61, 89-101.

Veerman, M. M., Brenner, E., \& Smeets, J. B. J. (2008). The latency for correcting a movement depends on the visual attribute that defines the target. Experimental Brain Research, 187, 219-228.

Warren, W. H., Young, D. S., \& Lee, D. N. (1986). Visual control of step length during running over irregular terrain. Journal of Experimental Psychology: Human Perception and Performance, 12(3), 259-266.

Weerdesteyn, V., Nienhuis, B., Hampsink, B., \& Duysens, J. (2004). Gait adjustments in response to an obstacle are faster than voluntary reactions. Human Movement Science, 23, 351-363.

Weerdesteyn, V., Nienhuis, B., Mulder, T., \& Duysens, J. (2005). Older women strongly prefer stride lengthening to shortening in avoiding obstacles. Experimental Brain Research, 161, 39-46.

Wolpert, D. M., Ghahramani, Z., \& Jordan, M. I. (1995). An internal model for sensorimotor integration. Science, 269, 1880-1882. 